\title{
The Coplanar Family of Bis(nitrotriazoles) Tetrazine and Oxides Based as Energetic Compounds
}

\author{
Jin $\mathrm{Xu}^{1}$, Jinting $\mathrm{Wu}^{1}$, Yuhe Jiang ${ }^{1}$, Lian Zeng ${ }^{1}$, Wei $\mathrm{Li}^{2}$, Hongbo $\mathrm{Li}^{1}$, and Jianguo Zhang ${ }^{3}$ \\ ${ }^{1}$ Southwest University of Science and Technology \\ ${ }^{2}$ China Academy of Engineering Physics \\ ${ }^{3}$ Beijing Institute of Technology
}

May 5, 2020

\begin{abstract}
Abstract: Searching for energetic materials with balanced detonation performance and sensitivity is the enduring ambition in the evolution of high energy density materials (HEDMs). The coplanar molecular structure of energetic compound has a powerful impact on performance. Herein, the novel compounds of bis(nitrotriazoles) tetrazine (BNTT) was designed and investigated by density functional theory(DFT) method. However, the coplanar BNTT's oxides would a highlight of molecular design with good balance between superior performance with acceptable sensitivities. Results show that all these designed compounds possess high densities, positive heats of formation, remarkable detonation performance, and acceptable impact sensitivity. In particular, B1-3 possess higher density $(\rho=1.97 \mathrm{~g} \cdot \mathrm{cm}-3)$ and exhibits the better balance between detonation performance $(\mathrm{Q}=1779.83 \mathrm{cal} \cdot \mathrm{g}-$ $1, \mathrm{D}=9.48 \mathrm{~km} \cdot \mathrm{s}-1, \mathrm{P}=42.01 \mathrm{GPa})$ and sensitivity $(\mathrm{h} 50 \%=28 \mathrm{~cm})$ than RDX. The theoretical study offer that all novel compounds possess acceptable sensitivity. It may be seen as the potential candidates of HEDMs.
\end{abstract}

\section{Hosted file}

Main document.docx available at https://authorea.com/users/298689/articles/428198-thecoplanar-family-of-bis-nitrotriazoles-tetrazine-and-oxides-based-as-energetic-compounds 